
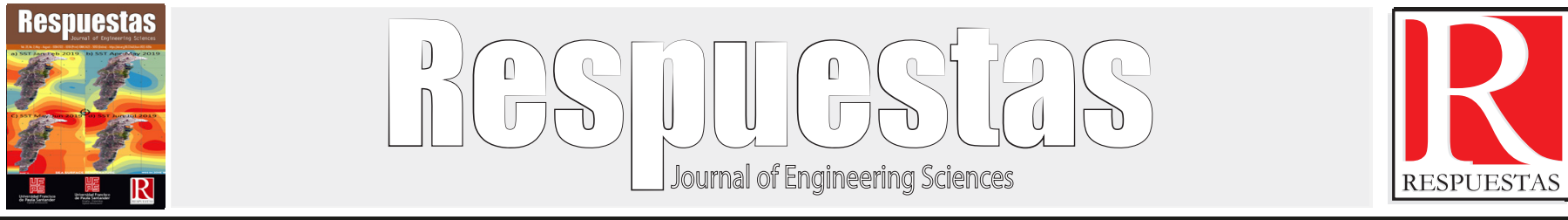

Original Article

https://doi.org/10.22463/0122820X.1929

\title{
Evaluación comparativa de un método de doma humanitaria y uno tradicional para el caballo de silla colombiano
}

Benchmarking of a method of dressage humanitarian and one for traditional saddle colombian horse

Johann Fernando Hoyos-Patiño ${ }^{1 *}$, Juan Carlos. Suarez-Salazar², Gloria Elena Estrada-Cely ${ }^{3}$

1 *Mg. Sistemas Sostenibles, Universidad Francisco de Paula Santander-Ocaña, jfhoyosp@ufpso.edu.co, Orcid: 0000-0002-0377-4664, Ocaña, Colombia.

2Ph.D. Biologia, Universidad de la Amazonía-Florencia, juansuarez1@gmail.com, Orcid:0001-5928-1837 Florencia, Colombia

3PhD. Bioética, Universidad de la Amazonia, Florencia, gestmvz@gmail.com, Orcid: 0000-0003-0576-4432 Florencia, Colombia

Como citar: J. Hoyos-Patiño, J. Suarez-Salazar y G. Estrada-Cely, "Evaluación comparativa de un método de doma humanitaria y uno tradicional para el caballo de silla colombiano". Respuestas, vol. 25, no. 1, pp. 159-167, 2020.

Received on February 07, 2020; Approved on April 5, 2020

\begin{tabular}{ll}
\hline RESUMEN \\
\hline Palabras clave: & La presente investigación tuvo como propósito la comparación de dos métodos de doma: el tradicional y \\
Bienestar animal, & el humanitario, evaluando sus efectos sobre parámetros conductuales y fisiológicos en 32 potros Criollos \\
caballo, & Colombianos, distribuidos equitativa y aleatoriamente para cada tipo de doma. El estudio comportamental \\
doma, & se desarrolló mediante el método observacional de registro continuo de tiempo pre y post doma, a partir del \\
etograma, & seguimiento de cuatro parámetros actitudinales o conductuales en los especímenes, que fueron: atención, \\
humanitaria, & desconfianza, agresividad y obediencia; y el fisiológico fue desarrollado a partir del seguimiento de la \\
potro, & frecuencia cardiaca, respiratoria, temperatura rectal, glucosa, leucocitos, linfocitos, monocitos y hemoglobina. \\
tradicional. & El análisis de los resultados demostró que aunque por ambos métodos se obtienen éxito en el proceso de \\
& doma, mediante la humanitaria se producen potros dóciles y relajados, menos expuestos a la presencia de \\
& enfermedades, mientras en la tradicional, potros temerosos y asustadizos.
\end{tabular}

\begin{tabular}{ll}
\hline ABSTRACT \\
\hline Keywords: & $\begin{array}{l}\text { The present research was aimed at comparing two methods of taming: the traditional and humanitarian, } \\
\text { evaluating its effects on behavioral and physiological parameters of } 32 \text { Colombian creole foals, distributed } \\
\text { equally and randomly for each type of dressage. The behavioral study was conducted by the observational } \\
\text { method of continuous recording of pre and post dressage time monitoring from four attitudinal or behavioral }\end{array}$ \\
Animal welfare, & parameters in the specimens, which were: attention, suspicion, aggression and obedience; and the \\
horse, & physiological study was developed from monitoring heart rate, respiratory rate, rectal temperature, glucose, \\
dressage, & leukocytes, lymphocytes, monocytes and hemoglobin. The analysis of the results showed that although both \\
ethogram, & methods succeed in taming process are obtained by humanitarian docile ponies and relaxed, less exposed to \\
humanitarian, & the presence of disease, while in traditional, fearful and skittish colts they occur..
\end{tabular}

\section{Introducción}

El uso del caballo como medio de transporte, militar y deportivo es conocido y desarrollado desde tiempos remotos [1], pero desde finales del siglo XX el hombre ha encomendado al caballo nuevas tareas como la de servir de apoyo a terapias para la recuperación de condiciones físicas o sicológicas de las personas.

Colombia es reconocida internacionalmente por sus ejemplares equinos, incluyendo mulares y asnales, de exposición, deporte y trabajo, equino de paso fino, trote, trocha o galope, ejemplar de coleo, carreras o tiro y mular y asnal de labor, que suman una población de 1'451.085 asentadas principalmente en los departamentos de Antioquia (10,61\%), Tolima (8,15\%), Cundinamarca (7,52\%), Córdoba (7,36\%), Casanare (5,69\%) y Cauca $(5,63 \%)$, es en estas regiones en donde se concentra el $45,21 \%$ del censo equino nacional, y en el ámbito regional, el censo equino en el área metropolitana de Cúcuta es de 1.835, siendo en la municipalidad de Villa del Rosario de 552 y en Pamplona de 631 [2]. 
El Caballo Criollo Colombiano, se caracteriza por su sensibilidad, brío, velocidad y suavidad; poseedor de un fenotipo único, elegante, noble, con movimientos bien definidos, que le permite a su montador gozar de gran quietud durante la monta; dichos rasgos son obtenidos no sólo por una adecuada dotación genética, sino además por un oportuno y acertado proceso de adiestramiento $[3]$.

La doma, entendida como un proceso de intervención antrópica, tiene como fin hacer que un animal sea capaz de obedecer órdenes específicas, que pueda interactuar con el hombre para el cumplimiento de una tarea específica. Al momento de acelerar dicho proceso usando la imposición, se disminuye la capacidad del caballo para asimilar la nueva información, activando sus instintos de defensa y huida [4], elevando los niveles de estrés expresados en el aumento de la frecuencia cardiaca, frecuencia respiratoria y niveles de la glucosa en sangre [5], pudiendo llegar a comprometer la sobrevivencia del animal.

Los procesos de doma tradicional impositiva, no permiten que el caballo desarrolle totalmente sus habilidades debido al miedo causado por la aplicación de algunas de sus técnicas, ante lo que se produce como respuesta, comportamientos indeseados y rechazo al contacto con el hombre [4] además de evidentes alteraciones fisiológicas que limitan su capacidad de adaptación y sobrevivencia; es por esto que las nuevas tendencias de doma no tradicional o sin violencia, usan como herramienta básica la etología, para formar un lenguaje de ayudas con el domador, que mejora el proceso creando un ambiente donde el caballo puede desarrollar sus actitudes de cría, además de facilitar la relación con el hombre [4].

Algunas de las consecuencias negativas de los sistemas de doma tradicional se describen por Reyna [6], en su trabajo titulado "La doma india de la Pampa Argentina, aplicada al caballo criollo Casanareño", en el que se referencia la crueldad en la aplicación de los métodos de doma tradicional empleados en los caballos del llano Colombiano y en algunas zonas de las Pampas Argentinas, donde el uso de la fuerza es la principal herramienta de disuasión para lograr los objetivos; por otra parte, Prado 2009 deja de manifiesto que los procesos de doma impositivos y violentos, producen vicios y alteraciones en el comportamiento de los equinos, mermando el desempeño y generando descartes prematuros [7].
Tradicionalmente en el país, el sistema de doma más frecuentemente aplicado, corresponde al de doma impositiva o por reforzamiento negativo, en el que se busca el adiestramiento del animal a través de la fuerza, el temor y el dolor; dicho proceso vulnera de forma directa el bienestar de los animales, comprometiendo tanto la integridad de los manejadores, como la sobrevivencia del animal; es por esto que cada vez adquieren mayor relevancia sistemas alternativos de doma, en los que se reconsidera el animal como ser físico, sintiente y emocional, en el marco de una trato humanitario y amable [8], permitiendo superar las limitantes de los sistemas de doma impositiva, a la vez de procurar transformaciones culturales y éticas hacia el respeto por lo vivo.

El sistema de doma no tradicional supone un mayor interés sobre el bienestar de los equinos, en consideración a las repercusiones fisiológicas, etológicas y éticas del proceso, por lo que se requiere una inmediata organización, comprobación y validación científica del método, de manera que se permita un impacto real en las poblaciones humanas, además de garantizar el éxito del mismo.

Los nuevos sistemas de doma no tradicional, caracterizados por la ausencia de violencia y la consideración de los animales como seres sintientes y emocionales [9], han sido lentamente introducidos en el país sin mayores impactos sobre el sistema, debido a la ausencia de evidencias científicas que permitan demostrar su efecto y ventajas comparativas y por ende, bajo nivel de confiabilidad y aplicación por parte de los domadores, por lo que resulta importante la necesaria identificación de los efectos conductuales y fisiológicos comparativos en la aplicación de un sistema de doma humanitaria versus un sistema de doma tradicional en el caballo de silla colombiano.

\section{Materiales y métodos}

\section{Área de estudio}

El estudio fue desarrollado en tres criaderos equinos del área metropolitana de la ciudad de Cúcuta y una pesebrera del municipio de Pamplona.

El área metropolitana de Cúcuta es una entidad administrativa creada por la ordenanza número 40 del 3 de enero de 1991 y puesta en funcionamiento por el decreto 508 del 3 de julio de ese mismo año. Se encuentra ubicada en la región oriental del departamento de Norte de San- 
tander. Su núcleo o municipio sede es Cúcuta, a su vez capital del departamento, e incluye los municipios circundantes de Los Patios, Villa del Rosario, El Zulia, San Cayetano y Puerto Santander [10].

El municipio de Pamplona, se localiza geográficamente en la Cordillera Oriental de los Andes Colombianos, a una altitud de $2200 \mathrm{msnm}$, en la zona suroccidental el departamento de Norte de Santander. Su extensión territorial es de $1.176 \mathrm{~km} 2$ y su temperatura promedio de 16 ${ }^{\circ} \mathrm{C}$. Limita al norte con Pamplonita, al sur con Cácota y Chitagá, al oriente con Labateca y al occidente con $\mathrm{Cu}-$ cutilla [11].

\section{Especímenes de estudio}

Para el desarrollo de la investigación se evaluó un grupo de 32 potros Criollos de Silla Colombiano, en edad de doma entre los 30 y 36 meses, con los que se conformaron dos grupos de trabajo de 16 potros distribuidos para los procesos de doma (tradicional y humanitaria), de manera aleatoria.

Los especímenes se evaluaron clínica y etológicamente con el fin de evitar la inclusión de animales con patologías que afectaran la validez de la información.

\section{Levantamiento de datos comportamentales.}

Los 32 potros seleccionados para el estudio, fueron inspeccionados clínicamente por un Médico Veterinario para la determinación de su estado de salud y condiciones para iniciar el proceso de doma. Posteriormente, se realizó la diagramación de la tabla de registro conductual; en cada registro se especificó la ubicación (lugar del criadero o pesebrera), humedad relativa promedio (HR), temperatura (T) y m s.n.m), tipo de doma, sexo, edad, color, modalidad, tipo de nutrición, lugar de permanencia, como información principal.

\section{Levantamiento de parámetros fisiológicos.}

Las variables fisiológicas de frecuencia cardiaca (FC), frecuencia respiratoria (FR), Temperatura (T), Glucosa, Lleucocitos (WBC), Linfocitos (Lym\#), Monocitos (Mon\#) y Hemoglobina (HGB), se midieron antes y después de cada sesión de entrenamiento.

Temperatura: su lectura fue realizada por vía rectal con el uso de termómetro Clínico Digital Unitest de punta flexible, previamente lubricado que se introdujo durante 3 a 5 minutos, dependiendo de la sensibilidad del sensor.

Frecuencia Cardiaca: fue identificada medio de auscultación con estetoscopio; el campo de auscultación del equino se encuentra delimitado cranealmente por el margen caudal de la escápula y la musculatura del hombro, dorsalmente por la musculatura del lomo, caudalmente se extiende hasta la altura de la tuberosidad coxal y ventralmente hacia el codo [12].

Frecuencia respiratoria: fue registrada mediante observación de los movimientos del tórax, flancos o el de los ollares. También se realizó acercándose al caballo, colocando la mano en los ollares para notar la salida de aire y contar las respiraciones por minuto. Para la auscultación directa con fonendoscopio, se ubicó la campana de auscultación en los primeros anillos traqueales a la altura de la laringe, que se pueden palpar en la zona ventral del cuello. En un caballo sano es de 8 a $20 \mathrm{rpm}$ y en los potros de 20 a $30 \mathrm{rpm}$ [12].

Muestra sanguínea: la muestra de sangre fue tomada con aguja calibre $(16,18$ o 21$)$ de la vena yugular, previa des-infección de la zona; obteniendo un total de $5 \mathrm{ml}$ por animal, que fue depositado en tubo con solución anticoagulante de EDTA (Vacutainner tapa lila), completamente rotulado, homogenizado suavemente y refrigerado para su envío al laboratorio donde se analizó con la maquina Mindray Analizador automático para hematología Bc-2800vet. Los datos de variables hematológicas que se evaluaron para el estudio fueron: WBC, Lym\#, Mon\#, Lyn\%, Mon\% y hemoglobina, y glicemia. Para la medición de la glicemia se utilizó un glucómetro portátil Accu-Chek Active.

\section{Análisis estadístico}

El desarrollo de la investigación se planteó a través de un diseño de parcelas al azar con 16 animales por tratamiento considerando como unidad experimental a cada uno de los individuos que conformaron el experimento. Para el análisis de los datos, se consideraron como tratamientos los tipos de doma (Doma Tradicional y Doma humanitaria) y como variables de respuesta tanto a las observaciones conductuales desarrolladas sobre cada animal, como a sus respuestas fisiológicas. 
Se realizaron correlaciones para determinar el nivel de relación entre las variables de conducta y fisiológicas de respuestas, en cada uno de los tipos de doma. Para el análisis exploratorio multivariado se utilizó el paquete estadístico el paquete $\mathrm{R}$ versión 2.15 [13], mediante la plataforma para análisis estadísticos R Commander [14] basado en el paquete FactoMineR [15].

\section{Implicaciones éticas y bioéticas}

En correspondencia con Estrada y Parra [16]; las implicaciones éticas y bioéticas en general, suponen dos tipos de consideracio-nes particulares, las de tipo intraespecífico, dentro de las que se estructuran las relaciones entre los seres humanos o personas, reconocidas por diversos autores del campo de la Bioética como bioética intraespecífica, microbioética o bioética deontológica, basada en el principio de igualdad y cuya prima face de responsabilidad reposa en el respeto a la autonomía y la no maleficencia; y las de tipo interespecífico, constituida para las relaciones establecidas entre los seres humanos y las demás especies vivientes, basadas en el principio de desigualdad y guiadas por los principios de justicia y beneficencia desde la premisa del principio de responsabilidad, dado el superior carácter del ser humano en su condición única de ético y moral. [17] - [21].

La totalidad de los preceptos anteriormente señalados, además de los lineamientos inter e intraespecíficos, establecidos por autores de relevancia internacional para el tema como Cardozo, Martínez, Rodriguez y Lolas [22], estructuran los cuestionamientos de análisis establecidos por el Comité de Ética, Bioética y Bienestar Animal de la Facultad de Ciencias Agropecua-rias de la Universidad de la Amazonía, por lo que la pre-sente investigación contó con el correspondiente Aval, según Acta 26 del 6 de octubre de 2015.

\section{Resultados}

\section{Respuesta conductual de los especímenes}

El estudio comportamental se desarrolló mediante el método observacional de registro continuo de tiempo para la etapa inicial, por la técnica ad libitum, con el fin de identificar los patrones conductuales de los especímenes en condiciones normales. Su instauración se realizará previa al proceso de doma.

Los especímenes objeto de estudio al momento de la investigación promediaban una edad de 32 meses, con igual manejo de dieta y ración, compuesta de pasto verde, heno suplementados con concentrado y características similares de permanencia en pesebrera y potrero.

Las condiciones promedio de temperatura ambiental, humedad relativa y altitud en los sitios de muestreo promediaron: Área metropolitana de Cúcuta $28.13^{\circ} \mathrm{c} ; 62.89 \%$ y $250 \mathrm{msnm}$, Villa del rosario $30.37^{\circ} \mathrm{c}$; $55.5 \%$ y $285 \mathrm{msnm}$ y Pamplona $15.57^{\circ} \mathrm{c}$; $72 \%$ y $2340 \mathrm{msnm}$; el muestreo se realizó desde el mes de julio del 2015 al mes de febrero de 2016.

Para el etograma inicial o diagnóstico, los especímenes se observaron durante ocho días, una hora diaria, en los corrales y potreros donde retozaron; una vez desarrollado el etograma, se identificaron cuatro parámetros actitudinales o conductuales en los especímenes, en correspondencia con los reportados y fueron: atención, desconfianza, agresividad y obediencia. Para cada parámetro conductual se definieron comportamientos específicos y se clasificó su respuesta en escala de valor de 1 a 3, con la

\section{siguiente interpretación:}

1: nula: cuando no se presenta ninguna manifestación.

2: media: cuando el nivel de presentación de la conducta es irregular

3: alta: cuando el nivel de manifestación de la conducta es constante durante el tiempo de observación.

El parámetro conductual de Atención (ATE), fue interpretado como la actitud o capacidad para mantenerse perceptivo a un estímulo específico, sin mediación de manifestaciones de estrés o tensión. Los comportamientos identificados para esta conducta correspondieron a la posición de orejas, ojos y boca, acompañado con el nivel de soltura o rigidez del cuello, dorso y extremidades [23].

El parámetro conductual de Obediencia (OBE) se refiere al instinto gregario del caballo para establecer y mantener un orden jerárquico de los individuos en la manada. 
Corresponde a la respuesta instintiva natural de recono-cer y seguir un líder [23].

El parámetro conductual de Desconfianza (DES) fue in-terpretado como la razón por la cual, el potro toma dis-tancia ante todo lo desconocido. El caballo en un animal neófobo (se asusta de todo lo que le resulta nuevo). Todas estas actitudes son la suma de posición de orejas, ojos, boca; acompañado con el nivel de soltura o rigidez del cuello, dorso y extremidades [23].

El parámetro conductual de Agresividad (AGR), surge cuando se siente acorralado y su instinto lo obliga a defenderse manoteando, mordiendo, pateando o corcovean-do. Estas dos últimas reacciones obedecen a que en la mayoría de los casos el caballo es alcanzado desde su parte trasera, manteniendo al predador a distancia me-diante patadas, o corcoveando para tratar de derribarlo de su lomo en caso de haber alcanzado a saltar sobre él. Todas estas actitudes son la suma de posición de orejas, ojos, boca; acompañado con el nivel de soltura o rigidez del cuello, dorso y extremidades [23].

Los dos primeros parámetros, son concebidos como po-sitivos, en la medida en que son identificados como éxi-tos del proceso; y los dos segundos como negativos en la medida en que suponen riesgos para los manejadores y la integridad del potro.

Durante el proceso de doma, y dado que el objetivo de la investigación correspondió a la evolución del logro del objetivo etológico, se realizó seguimiento mediante registro continuo de comportamientos a través la técnica de registro anec-dótico. La finalidad de este seguimiento corresponde a la identificación de tiempos para el logro del objetivo en la doma, el éxito de presentación del comportamiento deseado y la presencia e intensidad de comportamientos anómalos.

Los datos se clasificaron para los dos tipos de doma, du-rante la fase previa y posterior a la misma (Tabla I).

Tabla I. Resumen parámetros conductuales

\begin{tabular}{|c|c|c|c|c|c|}
\hline FACTOR & NIVEL & FASE & DESCONFLANZA & AGRESIIIDAD & OBEDIESCIA \\
\hline \multirow{3}{*}{ Dama } & \multirow{2}{*}{ Humannitaña } & Predoma: & $2=0,02: \mathrm{B}$ & $1.25=0,09$ AB & $2,31=0,09$ \\
\hline & & Posidoma & $91+0,08$ C $1,18=0,02 \mathrm{~A}$ & $112=0,09 \mathrm{~A}$ & $2,66=0,09 \cdot c$ \\
\hline & Tradicional & Pre doma & $88 \div 008-B \quad 2=0,02-B$ & $=156=0.09 \mathrm{C}$ & $=2 ; 31 \_0,09-\mathrm{B}$ \\
\hline
\end{tabular}

Se identificó que para el parámetro conductual de Aten-ción, para los dos tipos de doma, en el proceso pre- do-ma los especímenes se muestran igualmente atentos sin indicación de comportamientos de tensión o alerta; sin embrago, para el proceso pos-domas, los especímenes sometidos a doma humanitaria registran altos niveles de atención, contrario a los especímenes sometidos a la doma tradicional.

Para el parámetro de Obediencia en el proceso pre-doma, los especímenes se mostraron igualmente obedientes; sin embargo, los sometidos a doma humanitaria, continua-ron registrando altos niveles de obediencia, mientras los sometidos a doma tradicional, disminuyeron en nivel de manifestación de tal conducta, asociado esto con un incremento en los comportamientos de luche y huida. 
Para el parámetro conducta de Desconfianza, los especí-menes vinculados a los dos procesos de doma registraron un nivel medio de desconfianza; sin embrago, posterior a la, la conducta disminuye en la humanitaria y aumenta en la tradicional.

Para el parámetro de agresividad, los animales regis-traron bajo nivel de respuesta durante la pre-doma, que disminuyó durante el proceso de doma, registrando un mayor descenso en la tradicional.

Vale la pena indicar que las disminuciones específicas de la agresividad en cada proceso, no suponen diferencias significativas, aunque sí la manera como fue obtenida, debido a que si bien el producto final (animal domado), se mostró poco agresivo en ambos casos, durante el pro-ceso, la doma tradicional registra un alto grado de violen-cia, sometimiento y respuestas agresivas de los animales, contrario a la doma humanitaria.

\section{Respuesta fisiológica de los especímenes}

Los datos se clasificaron para los dos tipos de doma, du-rante la fase previa y posterior a la misma (Tabla II).

Tabla II. Comparación de variable fisiológica

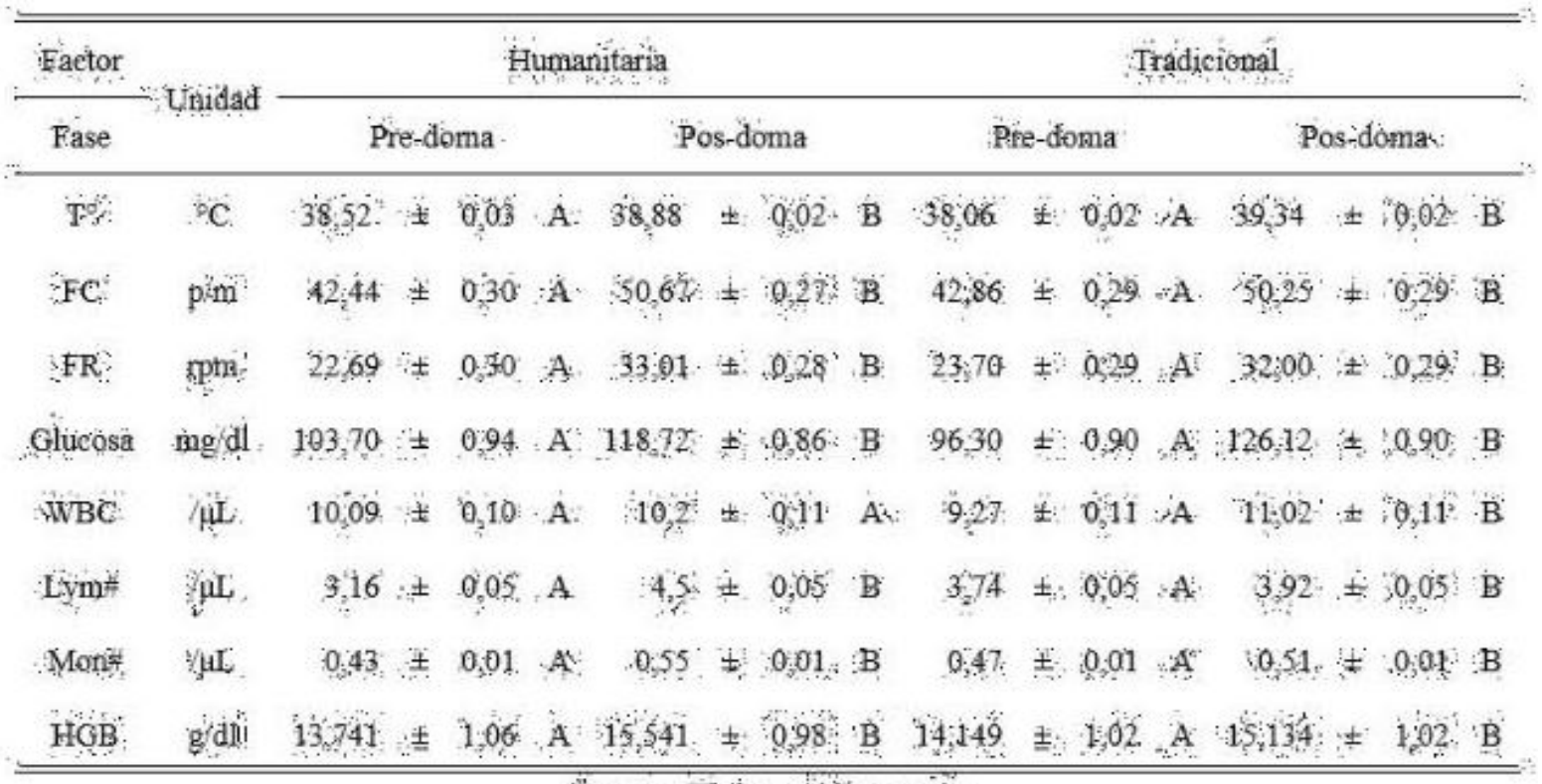

\section{F̈uente: Elaboración propid}

La temperatura rectal no reportó diferencias significati-vas para los procesos de doma; de manera similar, la fre-

cuencia cardiaca y respiratoria no reportaron diferencias significativas para los procesos de doma. La frecuencia cardiaca en el proceso de doma humanitaria, registró un incremento medio de 8.23 latidos por minuto, mientras en el proceso de doma tradicional, de 7.39; y la frecuencia respiratoria de 10,32 en el proceso de doma humanitaria y de 8.3 en el tradicional.

Los parámetros de glucosa, linfocitos, monocitos y hemoglobina, no reportaron diferencias significativas para los procesos de doma. La glucosa aumentó en $15.02 \mathrm{mg} /$ $\mathrm{dl}$ en la doma humanitaria y en $29.82 \mathrm{mg} / \mathrm{dl}$ en la doma tradicional; los linfocitos, que cumplen la función como segunda línea de defensa, después de los neutrófilos, presentaron un incremento de $1.34 / \mu \mathrm{L}$ en la doma humanitaria y de $0.18 / \mu \mathrm{L}$ en la tradicional; los monocitos presentaron un incremento de $0.12 / \mu \mathrm{L}$ en la doma humanitaria y de $0.04 / \mu \mathrm{L}$ en la tradicional; el incremento de la hemoglobina correspondió a $1.8 \mathrm{~g} / \mathrm{dl}$ en la doma humanitaria y a $9.85 \mathrm{~g} / \mathrm{dl}$ en la tradicional. 
Los glóbulos blancos indicaron diferencias significativas para el proceso de doma tradicional, con un aumentomedio de $1.75 / \mu \mathrm{L}$, mientras en la humanitaria fue de $0.11 / \mu \mathrm{L}$.

\section{Discusión}

\section{Respuesta conductual de los especímenes}

La manifestación de estados de tensión o alerta, asociados a la conducta de atención, identificados en los especímenes sometidos a doma tradicional, supone temor al domador y a los estímulos relacionados con su manejo.

Según Berrocal y López [24] "toda consecuencia de la conducta que sea recompensante $\mathrm{o}$, para decirlo más técnicamente, refor-zante, aumenta la probabilidad de nuevas respuestas"; por lo tanto, en la investigación, el proceso de doma hu-manitario constituye una manifestación de refuerzo po-sitivo, pues se busca el incremento de la frecuencia de un evento, por la consecución de un estímulo favorable, ra-zón por la cual, los especímenes registraron altos niveles de atención sin tensión; mientras la doma tradicional, podría identificarse como un proceso de reforzamiento negativo en el que se busca evitar un estímulo desagradable o castigo, por lo tanto registraron atención con tensión.

Para el parámetro de Obediencia en el proceso pre-doma, los especímenes se mostraron igualmente obedientes; sin embargo, los sometidos a doma humanitaria, continuaron registrando altos niveles de obediencia, mientras los sometidos a doma tradicional, disminuyeron en nivel de manifestación de tal conducta,

La disminución en la presentación del parámetro conductual de obediencia en especímenes sometidos a doma tradicional, permite suponer que, para el proceso de doma humanitaria, el domador es percibido por el animal, como un líder, lo cual facilita su manejo [4], [25] mientras en la tradicional, como un factor de estrés del que debe escapar, razón por la cual se registraron también mayores comportamientos de lucha o huida.

El registro de nivel medio para el parámetro conducta de Desconfianza, en los dos tipos de doma, se explican en consideración a lo novedoso que para los animales estos supone el proceso, ya que la novedad es una paradoja, en la medida en que atrae a la vez que provoca miedo

[26]. Posterior a la doma, la conducta disminuye la doma humanitaria y aumenta en la tradicional. Es decir, los animales sometidos a doma tradicional se muestran des-confiados, probablemente por el grado de asociación del proceso a un reforzamiento negativo.

Para el parámetro de agresividad, los animales registraron bajo nivel de respuesta durante la pre-doma, que disminuyó durante el proceso de doma, registrando un mayor descenso en la tradicional, lo anterior puede ser interpretado desde los patrones conductuales de especie, que como animal gregario, jerarquiza posiciones de grupo a partir de confrontaciones, ante lo que la imposición dolorosa de una jerarquía [9], [25], como lo establece el domador en la doma tradicional, suprime en mayor medida la respuesta de un animal.

\section{Respuesta fisiológica de los especímenes}

Aunque el parámetro de a temperatura rectal no reportó diferencias significativas para los procesos de doma, para la doma tradicional se registró incremento por encima del parámetro reportado para la especie, que se ubica entre $37.5-38.5^{\circ} \mathrm{c}$, [27], lo cual supone un mayor nivel de activación orgánica y gasto energético. De manera similar, la frecuencia cardiaca y respiratoria, que no reportaron diferencias significativas entre las domas, se situaron por encima de los rangos reportados para la especie que se ubica entre $36-40 \mathrm{p} / \mathrm{m}$ y de $8-20 \mathrm{rpm}$ [27]; [28], [12].

De manera similar, la frecuencia cardiaca y respiratoria no reportaron diferencias significativas para los procesos de doma, por encima de los rangos reportados para la especie que se ubica entre $36-40 \mathrm{p} / \mathrm{m}$ y de $8-20 \mathrm{rpm}$ [27]; [28], [12].

Los mayores incrementos de frecuencia cardiaca en la doma humanitaria se explican desde el referente de manejo del animal, pues el objetivo de respuesta conductual se obtiene mediante la repetición de un proceso específico acompañado de entrenamiento y no por mediación de un castigo. Es decir, en el proceso de doma humanitaria, cuando el animal no responde asertivamente, es alejado del proceso hasta que manifiesta su intención de retornar, de manera similar a su desarrollo conductual normal, en 
el que la madre ante un comportamiento negativo o desfavorable, aleja a la cría, [4], [25]. Durante las actividades translocadas, es decir, diferentes de las propias del proceso de doma, el potro generalmente corre por el picadero.

Los parámetros de glucosa, linfocitos, monocitos y hemoglobina, se encontraron dentro de las medias registradas para la especie que correspondieron a: de 90-144 mg/dl para glucosa según [29], de 1.021 a $5.896 / \mu \mathrm{L}$ para linfocitos; de 0 a $145 / \mu \mathrm{L}$ para monocitos y de $10.7-15.8 \mathrm{~g} /$ dl para hemoglobina [30], [31].

El mayor incremento de la glucosa en la doma tradicional, permite inferir una mayor respuesta de estrés durante el proceso de doma tradicional, en consideración a que la activación del eje hipotalámico-pituitario-adrenal (HPA) [32], reconocido como el mayor mediador de las respuestas endocrinas, liberando inicialmente catecolaminas como la adrenalina, que a su vez se encarga de inducir a nivel hepático procesos de glucogenólisis, fenómeno responsable de movilizar las reservas de energía para que el individuo pueda montar una respuesta física alternando el metabolismo de anabólico (almacenamiento) a catabólico (gasto) y suprimir los procesos no esenciales [31].

El estímulo causa que el hipotálamo libere Hormona Liberadora de Corticotropina (CRH), que pasa a través de los vasos Hipotálamo-Pituitaria anterior (HAP) a la pituitaria anterior, estimulándola a liberar Hormona Adrenocortico Trópica (ACTH) a la sangre [16].

La ACTH estimula que la corteza adrenal libere glucocorticoides a la sangre. Los glucocorticoides promuevan la glicogenólisis y la supresión de secreción de insulina en el hígado, esto aumenta la degradación de glicógeno a glucosa, aumentando los niveles de glucosa en la sangre (hiperglicemina); además promueva la liberación de dopamina, con lo que se eliminan o disminuyen las sanciones dolorosas [28].

Los linfocitos, que cumplen la función como segunda línea de defensa, después de los neutrófilos, presentaron un incremento de $1.34 / \mu \mathrm{L}$ en la doma humanitaria y de $0.18 / \mu \mathrm{L}$ en la tradicional,

El menor incremento de linfocitos en la doma tradicional se ha explicado explican manifestaciones de estrés crónico, crecimiento, lesiones o en caballos de carácter nervioso [33].

Los monocitos, para los tipos de doma, se ubicaron dentro de los rangos reportados como normales para la especie [34], por lo que no representan un parámetro para la determinación de estrés producto del proceso de doma.

El aumento presentado de la hemoglobina en la doma tradicional se explica porque el contenido de hemoglobina y la capacidad de transportar oxígeno aumenta en la sangre, debido al gasto fisiológico producto del ejercicio y en este caso de la exigencia física del proceso de doma tradicional, produciéndose la contracción del bazo que libera eritrocitos a la circulación [35].

La diferencia significativa de glóbulos blancos, mayores en la doma tradicional, se registra en concordancia con los argumentos de [36], quienes afirman que los leuco citos experimentan un aumento absoluto o relativo de Leucocitos durante situaciones estresantes del animal.

De igual manera, en la doma tradicional, al no aplicarse un trabajo progresivo y acorde al estado atlético y comportamental del potro, activa el sistema inmune predisponiéndolo a desequilibrios, a tal punto que esta misma relación puede ser más representativa que la concentración de cortisol como indicador de estrés [37].

\section{Conclusiones}

Con la aplicación de los dos sistemas de doma se obtie nen animales que permiten que el humano los monte y guíe; sin embargo, en la doma tradicional, los animales se muestran sumisos, excitables y temeroso a la manipula ción y contacto con el humano; mientras en la humanita ria, se muestran dóciles, relajados y con mayor capacidad para realizar diferentes oficios.

Los potros de cada grupo de doma presentaron parámetros fisiológicos dentro de los rangos normales para temperatura, frecuencia cardiaca y respiratoria, linfocitos, monocitos y hemoglobina, mostrando diferencia significativa para glucosa y leucocitos, que se registraron aumentadas en la doma tradicional, indicativo de mayor grado de respuesta de estrés.

De manera general es posible concluir que, tanto la doma 
tradicional como la humanitaria, resultan igualmente exitosas para el proceso de manejo del animal; sin em brago, con la doma tradicional, los animales se presentan excitables y temerosos, susceptibles a compromisos fisiológicos futuros, en consideración al aumento en la glicemia y activación del sistema inmune.

\section{Referencias}

[1] L . J . C o r r e d o r, "C a r a c t e ri z a c ión anatomofisiológica y estudio comportamental del caballo de monta para equinoterapia". (Tesis pregrado) Programa de medicina VeterinariaFacultad de Ciencias agropecuarias. Universidad de la Salle, Bogotá, Colombia, 2009.

[2] Instituto Colombiano Agropecuario-ICA. (2016, Mayo 12) Censo equino 2016. [En línea]: Disponible http://goo.gl/esoJcC.

[3] FedequinAS Colombia (2015, Junio 14) [En línea]. Disponible: http://goo.gl/8rGC22.

[4] J. Hoyos. "El lomo del caballo, segunda parte". Revista Universo Equino vol. 6, marzo-abril 2009.

[5] N. Martos and I. Ayala "El estrés en los equinos". Hospital Clínico Veterinario, Universidad de Murcia, pp. 121-127, 2003.

[6] L. Reyna. "La doma india de la pampa argentina, aplicada al caballo criollo casanareño". (Trabajo pregrado). Facultad de Zootecnia. Universidad de La Salle. Bogotá, Colombia, 2007.

[7] L. Prado. "Doma racional sin violencia en equinos". (Trabajo pregrado). Escuela de ciencia y tecnóloga en recursos agrícolas y acuícolas. Universidad de Magallanes Chile, 2009.

[8] J. Hoyos. "Evaluación comparativa de la aplicación del Imprinting en potros Criollos Colombianos" Revista de la Facultad de Ciencias Agropecuarias. Universidad de la Amazonia, vol.8 no 2, pp. $62-67.2008$.

[9] LEY 1774 de 2016. (2016, Enero 6). Congreso [18] L. Beauchamp, and F. Childress, Principios de Colombia. Diario oficial, Bogotá. [En línea]. de Ética Biomédica. España: Masson. S.A, pp. Disponible:http://goo. gl/G56ZbE. 522, 1999.

[10] Alcaldía de San José de Cúcuta-Norte de Santander. 2016. Si se puede progresar. [en línea] Disponible: http://goo.gl/Z6oEs8.

[11] Alcaldía del Municipio de Pamplona-Norte de Santander. 2016. El cambio en nuestras manos. [en línea] Disponible:http://goo.gl/LlMhz5.

[12] P.A. Guerrero, L. Portocarrero, C. A. Mutis, and J. Ramírez. "Determinación de frecuencia cardiaca, frecuencia respiratoria, lactato deshidrogenasa, creatinkinasa y ácido láctico en caballos durante competencia de salto en la Sabana de Bogotá". Rev. Med. Vet. n.17, pp. 3752, 2009.

[13] R. Development and R. A. Core. Language and Environment for Statistical Computing, R Foundation for Statistical Computing, Viena, Austria. 2012. [en línea]. Disponible: http:// www.Rproject.org.

[14] J. Fox. "The R Commander: A basicstatistics graphical user interface to R'. Journal of Statistical Software, vol. 19 no 9, pp 1-42, 2005.

[15] F. Husson, S. Lê, and J. Mazet. FactoMineR: Factor Analysis and Data Mining with R. package version 2.4.0. (programa de cómputo). 2007. Disponible: http://factominer.free.fr/.

[16] G. E. Estrada, and J. P. Parra. "Implicaciones éticas y bioéticas en la investigación científica”. Revista Ces de Medicina Veterinaria y Zootecnia,vol. 11 no 2, pp.115-118, 2016. 
[17] H. Engelhardt. Los fundamentos de la Bioética. Barcelona: Paidos, pp.545, 1995.

[19] J. Carmona, Macrobioética. Colección Bios y Ethos. Bogotá: Kimpres. Universidad el Bosque, pp, 13, 1999.

[20] G.Cely, G. El horizonte bioético de las ciencias: Reflexiones para elaborar una ecoética. Bogotá: Fundación Cultural Javeriana, pp. 89-91, 2002. and J. Ramírez.

[21] C. Beorlegui. Ética animal e idea de persona. cardiaca, frecuencia respiratoria, lactato Madrid: Universidad Pontifica Comillas, pp 14, deshidrogenasa, creatinkinasa y ácido láctico 2005.

[22] C. A. Cardozo., C. Martínez., E. Rodriguez, and F. Lolas. El animal como sujeto experimental, aspectos técnicos y éticos. Chile: Andros impresores, pp. 288, 2007.

[23] J. F. Hoyos. "Adiestramiento etológico del Austria. 2012. [en línea]. Disponible: http:// caballo-sistema de doma sin violencia para el www.Rproject.org. caballo de paso colombiano". Revista Universo

[24] B. Berrocal., and E, López. Trabajo de of Statistical Software, vol. 19 no 9, pp 1-42, psicología: Skinner. España: Ministerios de 2005. educación, cultura y deporte, 2008.

[25] M. Roberts. De mis manos a las tuyas. Factor Analysis and Data Mining with R. package EE.UU: Solvang, CA: Editorial Tutor, 2004. version 2.4.0. (programa de cómputo). 2007. Disponible: http://factominer.free.fr/.

[26] T. Grandin., and J. Mark La genética del comportamiento animal. Colorado: State University, 1998.
[27] B. Ramírez. Manual de semiología clínica vol. 11 no 2, pp.115-118, 2016. veterinaria. Ciencias agropecuarias. Manizales, Colombia: Editorial Universidad de Caldas, 2005.

[28] J. Gutiérrez., and C. González. Fisiología aplicada a la veterinaria y zootecnia. Manizales, Colombia: Editorial Universidad de Caldas, pp. 180, 2005.

[29] M. Ojeda, "Concentraciones sanguíneas de glucosa y lactato post ejercicio estandarizado en equinos F.S.C de diferente aptitud competitiva". (Tesis pregrado). Escuela de Medicina Veterinaria. Universidad Santo Tomás, Santiago, Chile, 1996.

[30] C. A. Castillo., M. Tobón., C. A. Cano., J. Mira., A. P. Suárez., and E. M. Vásquez Valores hematológicos en caballos criollos colombianos del Valle de Aburrá. Colombia: Corporación Universitaria Lasallista, 2010.

[31] C.M. Solar. "Cambios en indicadores sanguíneos de bienestar animal en caballos criollo chileno sometidos al ejercicio de rodeo". (Tesis pregrado). Facultad de ciencias veterinarias instituto de ciencia animal. Universidad austral de Chile. Valdivia-Chile: 2012.

[32] F. Duval., F. González., and H. Rabia. "Neurobiología del estrés". Revista chilena de neuro-psiquiatría, vol. 48 no 4, pp. 307-318., 2010.

[33] A. García., L.F Castejón., L.F de la Cruz., M. Gónzalez., M.D Murillo., and G. salido. Fisiología Veterinaria. España: Mc Graw - Hill, pp. $1074,1995$.

[34] M. Benjamin. Manual de Patología Clínica en Veterinaria. México: Limusa, pp. 120, 1991. 
[35] J. N. Ussa., and J.A. Salgado. "Determinación de hematocrito (hto), proteínas plasmáticas totales (ppt) y albumina (alb) en caballos de salto antes y después de cada entrenamiento en Bogotá". (Tesis pregrado). Facultad de ciencias agropecuarias programa de medicina veterinaria. Universidad de la Salle, Bogotá, 2009.

[36] D. A. Bohórquez., and N. J. Duque, "Valores hematológicos en yeguas paso fino colombiano y sus variaciones con ejercicio, en los municipios de Tabio y Cajica Cundinamarca". Facultad de Ciencias Agropecuarias. Programa de Medicina Veterinaria. Universidad de la Salle, Bogotá, 2010 .

[37] C. Stull., and A. Rodiek, "Physiological responses of horses to 24 hours of transportation using a commercial van during summer conditions". Journal of Animal Science, vol,78, pp. 1458-1466, 2000. 